\title{
DERIVED EQUIVALENCE CLASSIFICATION OF SYMMETRIC ALGEBRAS OF POLYNOMIAL GROWTH
}

\author{
THORSTEN HOLM \\ Institut für Algebra, Zahlentheorie und Diskrete Mathematik, Fakultät für Mathematik und Physik, \\ Leibniz Universität Hannover, Welfengarten 1, 30167 Hannover, Germany \\ e-mail:holm@math.uni-hannover.de \\ http://www.iazd.uni-hannover.de/ tholm \\ and ANDRZEJ SKOWROŃSKI \\ Faculty of Mathematics and Computer Science, Nicolaus Copernicus University, Chopina 12/18, \\ 87-100 Toruń, Poland \\ e-mail: skowron@mat.uni.torun.pl
}

(Received 9 November 2009; accepted 18 June 2010; first published online 8 December 2010)

\begin{abstract}
We complete the derived equivalence classification of all symmetric algebras of polynomial growth, by solving the subtle problem of distinguishing the standard and nonstandard nondomestic symmetric algebras of polynomial growth up to derived equivalence.
\end{abstract}

2010 Mathematics Subject Classification. 16G10, 18E30, 16D50, 16 G60.

1. Introduction and the main result. Throughout the paper, $K$ will denote a fixed algebraically closed field. By an algebra is meant an associative, finite-dimensional $K$-algebra with an identity. For an algebra $A$, we denote by $\bmod A$ the category of finitedimensional right $A$-modules and by $D$ the standard duality $\operatorname{Hom}_{K}(-, K) \operatorname{on} \bmod A$. An algebra $A$ is called self-injective if $A_{A}$ is an injective $A$-module, or equivalently, the projective $A$-modules are injective. Prominent classes of self-injective algebras are formed by the Frobenius algebras $A$ for which there exists an associative, nondegenerate, $K$-bilinear form $(-,-): A \times A \rightarrow K$, and the symmetric algebras $A$ for which there exists an associative, symmetric, nondegenerate, $K$-bilinear form $(-,-): A \times A \rightarrow K$. By the classical theorems of Nakayama [30,31], an algebra $A$ is Frobenius (respectively, symmetric) if and only if $A \cong D(A)$ in $\bmod A$ (respectively, as $A$ - $A$-bimodules). We also mention that every self-injective algebra $A$ is Morita equivalent to a Frobenius algebra, namely to its basic algebra. Moreover, for every algebra $B$, the trivial extension $\mathrm{T}(B)=B \ltimes D(B)$ of $B$ by the $B-B$-bimodule $D(B)$ is a symmetric algebra, and $B$ is a factor algebra of $\mathrm{T}(B)$. It follows also from a result of Nakayama [31] that the left socle and the right socle of a self-injective algebra $A$ coincide, and we denote them by $\operatorname{soc}(A)$. Two self-injective algebras $A$ and $\Lambda$ are said to be socle equivalent if the factor algebras $A / \operatorname{soc}(A)$ and $\Lambda / \operatorname{soc}(\Lambda)$ are isomorphic.

According to the remarkable Tame and Wild Theorem of Drozd [12], the class of (finite-dimensional) $K$-algebras over $K$ may be divided into two disjoint classes. One class consists of the tame algebras for which the indecomposable modules occur, in each dimension $d$, in a finite number of discrete and a finite number of one-parameter families. The second class consists of the wild algebras for which the representation 
theory comprises the representation theories of all finite-dimensional algebras over $K$ (see [37, Chapter XIX]). Hence a classification of finite-dimensional modules is only feasible for tame algebras. More precisely, following Drozd [12], an algebra $A$ is said to be tame if for any positive integer $d$, there exists a finite number of $K[x]-A$ bimodules $M_{i}, 1 \leq i \leq n_{d}$, which are finitely generated and free as left $K[x]$-modules $(K[x]$ is the polynomial algebra in one variable over $K$ ), and all but finitely many isomorphism classes of indecomposable modules of dimension $d$ in $\bmod A$ are of the form $K[x] /(x-\lambda) \otimes_{K[x]} M_{i}$ for some $\lambda \in K$ and some $i \in\left\{1, \ldots, n_{d}\right\}$. Let $\mu_{A}(d)$ be the least number of $K[X]-A$-bimodules satisfying the above condition for $d$. Then $A$ is said to be of polynomial growth (respectively, domestic) if there exists a positive integer $m$ such that $\mu_{A}(d) \leq d^{m}$ (respectively, $\mu_{A}(d) \leq m$ ) for all $d \geq 1$. Moreover, from the validity of the second Brauer-Thrall conjecture, $\mu_{A}(d)=0$ for all $d \geq 1$ if and only if $A$ is representation-finite (there are only finitely many isomorphism classes of indecomposable modules in $\bmod A$ ).

One central problem of modern representation theory is the determination of the module categories $\bmod A$ of tame self-injective algebras $A$. Recently, the module categories of all self-injective algebras of polynomial growth have been described completely. It has been proved by the second named author [38] that a nonsimple basic connected self-injective algebra $A$ is of polynomial growth if and only if $A$ is socle equivalent to an orbit algebra $\widehat{B} / G$, where $\widehat{B}$ is the repetitive category of an algebra $B$, being a tilted algebra of Dynkin or Euclidean type or a tubular algebra, and $G$ is an admissible infinite cyclic automorphism group of $\widehat{B}$. In particular, the Morita equivalence classification of the self-injective algebras of polynomial growth splits into two cases: the standard algebras whose basic algebras admit simply connected Galois coverings, and the remaining nonstandard algebras (see [39]). We refer to the survey article [40] for the Morita equivalence classification and the structure of module categories of the self-injective algebras of polynomial growth.

In this paper, we are concerned with the problem of derived equivalence classification of self-injective algebras of polynomial growth. For an algebra $A$, we denote by $D^{b}(\bmod A)$ the derived category of bounded complexes from $\bmod A$. Then two algebras $A$ and $\Lambda$ are said to be derived equivalent if the derived categories $D^{b}(\bmod A)$ and $D^{b}(\bmod \Lambda)$ are equivalent as triangulated categories. Since Happel's work [17] interpreting tilting theory in terms of equivalences of derived categories, the machinery of derived categories has been of interest to representation theorists. In [33] Rickard proved his celebrated criterion: two algebras $A$ and $\Lambda$ are derived equivalent if and only if $\Lambda$ is the endomorphism algebra of a tilting complex over $A$. Since a lot of interesting properties are preserved by derived equivalences (see Section 2), it is for many purposes important to classify classes of algebras up to derived equivalence, instead of Morita equivalence. In particular, for self-injective algebras the representation types introduced above are invariants of the derived category.

In [3, Theorem 2.2] Asashiba proved that the derived equivalence classes of connected representation-finite standard (respectively, nonstandard) self-injective algebras are determined by the combinatorial data called the types, and the derived equivalence classes of the standard and nonstandard representation-finite self-injective algebras are disjoint.

A complete derived equivalence classification of the representation-infinite domestic standard (respectively, nonstandard) symmetric algebras has been established in our joint papers with Bocian [10] (respectively, [11]). In [22] we completed the classification by showing that the derived equivalence classes of the standard and 
nonstandard representation-infinite domestic symmetric algebras are disjoint. In fact, we established in [22] the derived equivalence classification of all connected domestic symmetric algebras by bound quiver algebras.

A derived equivalence classification of the nondomestic standard (respectively, nonstandard) symmetric algebras of polynomial growth has been established in our joint papers with Białkowski [6] (respectively, [7]). This classification is complete up to certain scalar parameters occurring in the relations for which it seems intractable by current methods to decide for which scalars the corresponding algebras are derived equivalent.

The main open question in the derived equivalence classification of nondomestic symmetric algebras of polynomial growth has been to distinguish the standard algebras from the nonstandard algebras up to derived equivalence. This is subtle because the stable Auslander-Reiten quivers of all these algebras consist only of stable tubes (see $[32,39,40])$.

In this paper we solve this problem by proving the following main result and hence complete (up to the scalars mentioned above) the derived equivalence classification of the symmetric algebras of polynomial growth.

Main Theorem. Let $A$ be a standard self-injective algebra and $\Lambda$ be a basic, connected, nonstandard, nondomestic, symmetric algebra of polynomial growth. Then $A$ and $\Lambda$ are not derived equivalent.

In particular, it follows that the derived equivalence classes of the standard and nonstandard nondomestic symmetric algebras of polynomial growth are disjoint. For a list of explicit representatives of the derived equivalence classes, we refer to Section 4 below.

The crucial tool for proving the main theorem are the so-called Külshammer ideals defined by Külshammer in [25-28] for symmetric algebras of positive characteristic, which have been shown by Zimmermann [42] to be invariants of derived equivalences. These invariants are suitable for our purposes since the nonstandard nondomestic self-injective algebras of polynomial growth occur only in characteristics 2 and 3; see [9]. Moreover, in our proof of the Main Theorem we apply also a recent result of Al-Kadi [1] describing the dimensions of the second Hochschild cohomology spaces of the preprojective algebra of Dynkin type $\mathbb{D}_{4}$ and its unique proper deformation (in the sense of [5]).

In fact, the Main Theorem completes also the derived equivalence classification of tame symmetric algebras $A$ with periodic modules (all modules in $\bmod A$ without projective direct summands are periodic with respect to the action of the syzygy operator $\Omega_{A}$ ). Recall that $\Omega_{A}$ assigns to a module $M$ in mod $A$ the kernel of a minimal projective cover $P_{A}(M) \rightarrow M$. Since for the symmetric algebras $A$, the second syzygy $\Omega_{A}^{2}$ is the Auslander-Reiten translation $\tau_{A}=D \operatorname{Tr}$ (as functors on the stable module category $\underline{\bmod } A$ ), the class of tame symmetric algebras $A$ with $\Omega_{A}$-periodic module categories coincides with the class of tame symmetric algebras $A$ for which the stable Auslander-Reiten quiver $\Gamma_{A}^{S}$ consists only of stable tubes. It has been proved recently by Erdmann and Skowroński [13] that a nonsimple, basic, connected, symmetric algebra is tame with periodic modules if and only if $A$ is of one of the following forms: a representation-finite symmetric algebra, a nondomestic symmetric algebra of polynomial growth, or an algebra of quaternion type (in the sense of [13]). We refer to [13, Chapter VII] and [15, Section 5] (see also [40, Section 8]) for a Morita equivalence classification of algebras of quaternion type. The derived equivalence classification of 
all algebras of quaternion type has been established by the first author in [21, Section 5] (see also [15, Propositions 5.4 and 5.8]).

For basic background on the representation theory, we refer to the books $[4,36,37]$ and to the survey articles $[\mathbf{4 0}]$ and $[\mathbf{4 1}]$.

2. Invariants of derived equivalences of algebras. The aim of this section is to present some properties of algebras which are invariant under derived equivalences.

The following results of Rickard [35, Corollary 5.3] (symmetric case) and AlNofayee [2] (self-injective case) establish invariance of the classes of symmetric algebras and self-injective algebras under derived equivalences.

TheOREM 2.1. Let $A$ and $\Lambda$ be derived equivalent algebras. Then the following equivalences hold.

(i) $A$ is symmetric if and only if $\Lambda$ is symmetric.

(ii) $A$ is self-injective if and only if $\Lambda$ is self-injective.

Recall that the two algebras $A$ and $\Lambda$ are said to be stably equivalent if the stable module categories $\underline{\bmod } A$ and $\underline{\bmod } \Lambda$ (modulo projectives) are equivalent. The following result proved by Rickard in [34, Corollary 2.2] (see also [35, Corollary 5.5]) is fundamental for the study of stable and derived equivalences of self-injective algebras.

THEOREM 2.2. Let $A$ and $\Lambda$ be derived equivalent self-injective algebras. Then $A$ and $\Lambda$ are stably equivalent.

This together with the following theorem of Krause and Zwara [24] shows that the hierarchy of tame self-injective algebras is preserved by derived equivalences.

THEOREM 2.3. Let $A$ and $\Lambda$ be stably equivalent algebras. Then $A$ is tame (respectively, domestic, of polynomial growth) if and only if $\Lambda$ has the same property.

For a self-injective algebra $A$, denote by $\Gamma_{A}^{S}$ the stable Auslander-Reiten quiver of $A$, obtained from the Auslander-Reiten quiver $\Gamma_{A}$ of $A$ by removing the projective vertices and the arrows attached to them.

We have the following important consequence of Theorem 2.2.

Corollary 2.4. Let $A$ and $\Lambda$ be derived equivalent self-injective algebras. Then $\Gamma_{A}^{S}$ and $\Gamma_{\Lambda}^{s}$ are isomorphic as translation quivers.

For an algebra $A$, consider the enveloping algebra $A^{e}=A^{\text {op }} \otimes_{K} A$ of $A$. Recall that the category $\bmod A^{e}$ of finite-dimensional right $A^{e}$-modules is equivalent to the category of finite-dimensional $A-A$-bimodules. Moreover, $A$ is self-injective if and only if $A^{e}$ is self-injective. The algebra $A$ is a right $A^{e}$-module, via $a(x \otimes y)=x a y$ for $a \in A$, $x \in A^{\mathrm{op}}, y \in A$. We may then consider the Hochschild cohomology algebra

$$
H H^{*}(A)=\operatorname{Ext}_{A^{e}}^{*}(A, A)=\bigoplus_{i \geq 0} \operatorname{Ext}_{A^{e}}^{i}(A, A)
$$

of $A$, which is a graded commutative $K$-algebra with respect to the Yoneda product (see [16, Corollary 1]). We note that $H H^{0}(A)$ is isomorphic to the centre $Z(A)$ of $A$.

The following theorem proved by Rickard in [35, Proposition 2.5] (see also [18, Theorem 4.2] for a special case) shows that Hochschild cohomology yields invariants under derived equivalences. 
TheOrem 2.5. Let $A$ and $B$ be derived equivalent algebras. Then $H H^{*}(A)$ and $H H^{*}(B)$ are isomorphic as graded $K$-algebras.

COROLlary 2.6. Let $A$ and $B$ be derived equivalent algebras. Then the centres $Z(A)$ and $Z(B)$ are isomorphic as $K$-algebras.

We also note the following fact (see [18, Lemma III.1.2]).

THEOREM 2.7. Let $A$ and $B$ be derived equivalent algebras. Then the Grothendieck groups $K_{0}(A)$ and $K_{0}(B)$ are isomorphic.

3. Külshammer ideals. A prominent role in the proof of our main result is played by Külshammer ideals, introduced by Külshammer for symmetric algebras over algebraically closed fields of positive characteristic. These form a decreasing sequence of ideals of the centre of an algebra, and this sequence has been shown by Zimmermann [42] to be invariant under derived equivalences. We recall the definition of Külshammer ideals below; for more details on these invariants, we refer to [20, 23, 25-29].

Let $K$ be an algebraically closed field of characteristic $p>0$ and $A$ be a symmetric $K$-algebra, i.e. there exists an associative, symmetric, nondegenerate, $K$-bilinear form $(-,-): A \times A \rightarrow K$. For a $K$-subspace $M$ of $A$, denote by $M^{\perp}$ the orthogonal complement of $M$ inside $A$ with respect to the form $(-,-)$. Moreover, let $K(A)$ be the $K$-subspace of $A$ generated by all commutators $[a, b]:=a b-b a$, for any $a, b \in A$. For any $n \geq 0$, set

$$
T_{n}(A)=\left\{x \in A \mid x^{p^{n}} \in K(A)\right\} .
$$

Then, by [25-28], the orthogonal complements $T_{n}(A)^{\perp}, n \geq 0$, are ideals of the centre $Z(A)$ of $A$, called Külshammer ideals. They form a descending sequence

$$
Z(A)=T_{0}(A)^{\perp} \supseteq T_{1}(A)^{\perp} \supseteq T_{2}(A)^{\perp} \supseteq T_{3}(A)^{\perp} \supseteq \cdots .
$$

In fact, Külshammer proved in [29] that the equation $\left(\xi_{n}(z), x\right)^{p^{n}}=\left(z, x^{p^{n}}\right)$ for any $x, z \in Z(A)$ defines a mapping $\xi_{n}: Z(A) \rightarrow Z(A)$ whose image $\xi_{n}(Z(A))=T_{n}(A)^{\perp}$ is precisely the $n$th Külshammer ideal.

Then we have the following theorem proved recently by Zimmermann [42, Theorem 1] showing that the Külshammer ideals are derived invariants.

THEOREM 3.1. Let $A$ and $B$ be derived equivalent symmetric algebras over an algebraically closed field of positive characteristic $p$. Then there is an isomorphism $\varphi: Z(A) \rightarrow Z(B)$ of the centres of $A$ and $B$ such that $\varphi\left(T_{n}(A)^{\perp}\right)=T_{n}(B)^{\perp}$ for all nonnegative integers $n$.

Hence the sequence of Külshammer ideals gives new derived invariants, for symmetric algebras over algebraically closed fields of positive characteristic.

For using Külshammer ideals in the context of derived equivalence classifications, one has to be able to perform explicit computations with them and for this we 
shall later give explicit symmetrizing bilinear forms on the symmetric algebras under consideration.

4. Derived normal forms of nondomestic symmetric algebras of polynomial growth. In this section we present derived normal forms, i.e. representatives of the derived equivalence classes, of the connected nondomestic symmetric algebras of polynomial growth.

Consider the following families of bound quiver algebras (in the notation of [7]):

$\Lambda_{2}$ :

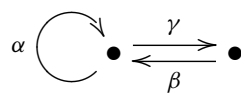

$\alpha^{2} \gamma=0, \beta \alpha^{2}=0, \gamma \beta \gamma=0, \beta \gamma \beta=0$, $\beta \gamma=\beta \alpha \gamma, \alpha^{3}=\gamma \beta$

$\Lambda_{3}(\lambda), \lambda \in K \backslash\{0,1\}:$

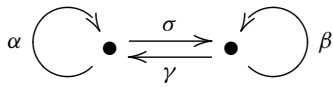

$$
\alpha^{4}=0, \gamma \alpha^{2}=0, \alpha^{2} \sigma=0,
$$

$\alpha^{2}=\sigma \gamma+\alpha^{3}, \lambda \beta^{2}=\gamma \sigma, \gamma \alpha=\beta \gamma$,

$$
\sigma \beta=\alpha \sigma
$$

$\Lambda_{5}$ :

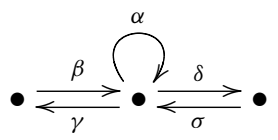

$$
\alpha^{2}=\gamma \beta, \alpha^{3}=\delta \sigma, \beta \delta=0, \sigma \gamma=0,
$$$$
\alpha \delta=0, \sigma \alpha=0, \gamma \beta \gamma=0, \beta \gamma \beta=0 \text {, }
$$$$
\beta \gamma=\beta \alpha \gamma
$$

$\Lambda_{9}$ :

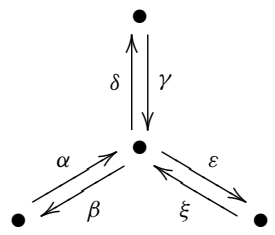

$\beta \alpha+\delta \gamma+\varepsilon \xi=0, \gamma \delta=0, \xi \varepsilon=0$, $\alpha \beta \alpha=0, \beta \alpha \beta=0, \alpha \beta=\alpha \delta \gamma \beta$
$\Lambda_{2}^{\prime}$ :

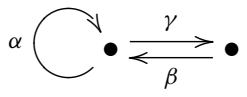

$\alpha^{3}=\gamma \beta, \beta \gamma=0$,

$\beta \alpha^{2}=0, \alpha^{2} \gamma=0$

$\Lambda_{3}^{\prime}(\lambda), \lambda \in K \backslash\{0,1\}:$

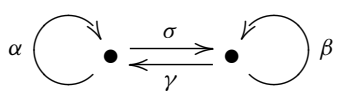

$\alpha^{2}=\sigma \gamma, \lambda \beta^{2}=\gamma \sigma$,

$\gamma \alpha=\beta \gamma, \sigma \beta=\alpha \sigma$

$\Lambda_{5}^{\prime}$ :

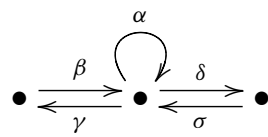

$\alpha^{2}=\gamma \beta, \beta \delta=0, \beta \gamma=0, \sigma \gamma=0$, $\alpha \delta=0, \sigma \alpha=0, \alpha^{3}=\delta \sigma$

$\Lambda_{9}^{\prime}$ :

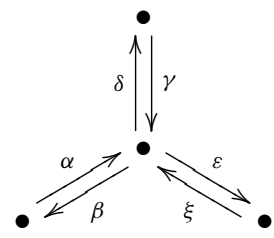

$\beta \alpha+\delta \gamma+\varepsilon \xi=0, \alpha \beta=0, \xi \varepsilon=0$, $\gamma \delta=0$ 
$A_{1}(\lambda), \lambda \in K \backslash\{0,1\}:$

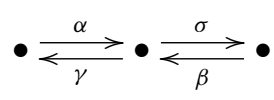

$\alpha \gamma \alpha=\alpha \sigma \beta, \beta \gamma \alpha=\lambda \beta \sigma \beta$,

$\gamma \alpha \gamma=\sigma \beta \gamma, \gamma \alpha \sigma=\lambda \sigma \beta \sigma$
$A_{4}$ :

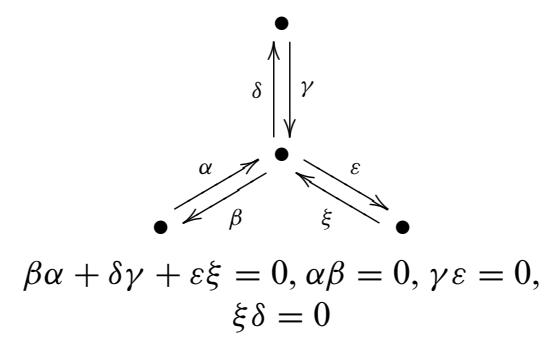
$\mathbb{D}_{4}$.

Note that the algebra $\Lambda_{9}^{\prime}$ is just the preprojective algebra of Dynkin type

It has been proved in [8, Theorems 1 and 2] and [9, Theorem 1.1] that

- $\Lambda_{2}$ and $\Lambda_{2}^{\prime}$ are symmetric algebras of tubular type $(3,3,3)$, and $\Lambda_{2} \cong \Lambda_{2}^{\prime}$ if and only if char $K \neq 3$. Moreover, $\Lambda_{2}$ and $\Lambda_{2}^{\prime}$ are socle equivalent.

- $\Lambda_{3}(\lambda)$ and $\Lambda_{3}^{\prime}(\lambda), \lambda \in K \backslash\{0,1\}$, are symmetric algebras of tubular type $(2,2,2,2)$ and $\Lambda_{3}(\lambda) \cong \Lambda_{3}^{\prime}(\lambda)$ if and only if char $K \neq 2$. Moreover, $\Lambda_{3}(\lambda)$ and $\Lambda_{3}^{\prime}(\lambda)$ are socle equivalent.

- $\Lambda_{5}$ and $\Lambda_{5}^{\prime}$ are symmetric algebras of tubular type $(2,4,4)$, and $\Lambda_{5} \cong \Lambda_{5}^{\prime}$ if and only if char $K \neq 2$. Moreover, $\Lambda_{5}$ and $\Lambda_{5}^{\prime}$ are socle equivalent.

- $\Lambda_{9}$ and $\Lambda_{9}^{\prime}$ are weakly symmetric algebras of tubular type $(3,3,3)$, and $\Lambda_{9} \cong \Lambda_{9}^{\prime}$ if and only if char $K \neq 2$. Furthermore, $\Lambda_{9}$ and $\Lambda_{9}^{\prime}$ are symmetric algebras if and only if char $K=2$. Moreover, $\Lambda_{9}$ and $\Lambda_{9}^{\prime}$ are socle equivalent.

- $A_{1}(\lambda), \lambda \in K \backslash\{0,1\}$, are symmetric algebras of tubular type $(2,2,2,2)$.

- $A_{4}$ is a symmetric algebra of tubular type $(3,3,3)$.

It follows also from [7, Theorem 2.1] that $\Lambda_{i}^{\prime}$ is a geometric degeneration of $\Lambda_{i}$ for $i \in\{2,5,9\}$, and $\Lambda_{3}^{\prime}(\lambda)$ is a geometric degeneration of $\Lambda_{3}(\lambda)$ for $\lambda \in K \backslash\{0,1\}$.

Consider also the following family of the trivial extensions of Ringel's tubular canonical algebras:

$\Lambda(2,2,2,2, \lambda), \lambda \in K \backslash\{0,1\}:$

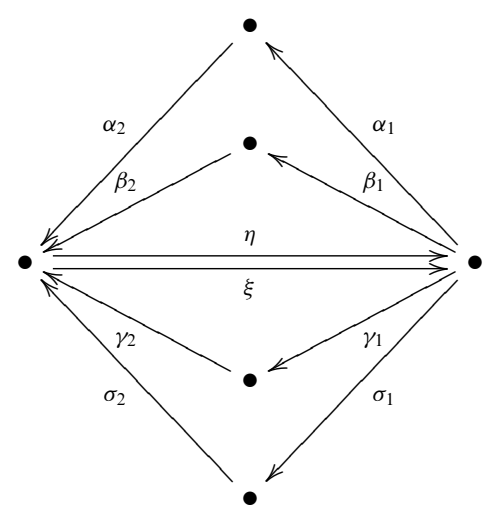

$\alpha_{1} \alpha_{2}+\beta_{1} \beta_{2}+\gamma_{1} \gamma_{2}=0, \alpha_{1} \alpha_{2}+\lambda \beta_{1} \beta_{2}+\sigma_{1} \sigma_{2}=0, \eta \alpha_{1}=0, \alpha_{2} \eta=0, \xi \beta_{1}=0, \beta_{2} \xi=$ $0, \eta \gamma_{1}=\xi \gamma_{1}, \gamma_{2} \eta=\gamma_{2} \xi, \eta \sigma_{1}=\lambda \xi \sigma_{1}, \sigma_{2} \eta=\lambda \sigma_{2} \xi$. 
$\Lambda(3,3,3)$ :

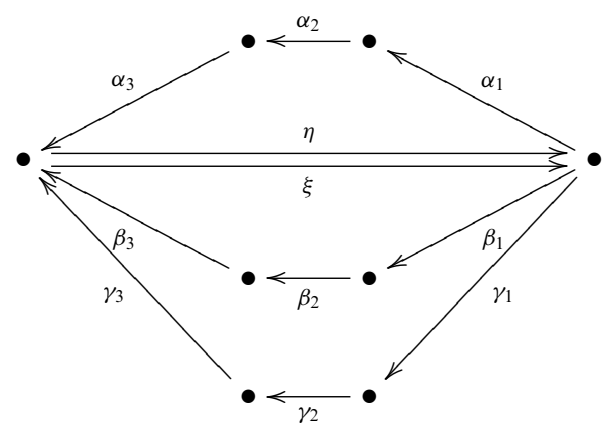

$\alpha_{1} \alpha_{2} \alpha_{3}+\beta_{1} \beta_{2} \beta_{3}+\gamma_{1} \gamma_{2} \gamma_{3}=0, \eta \alpha_{1}=0, \alpha_{3} \eta=0, \xi \beta_{1}=0, \beta_{3} \xi=0, \eta \gamma_{1}=\xi \gamma_{1}, \gamma_{3} \eta=$ $\gamma_{3} \xi, \alpha_{2} \alpha_{3} \xi \alpha_{1} \alpha_{2}=0, \beta_{2} \beta_{3} \eta \beta_{1} \beta_{2}=0, \gamma_{2} \gamma_{3} \eta \gamma_{1} \gamma_{2}=0$.

$\Lambda(2,4,4)$ :

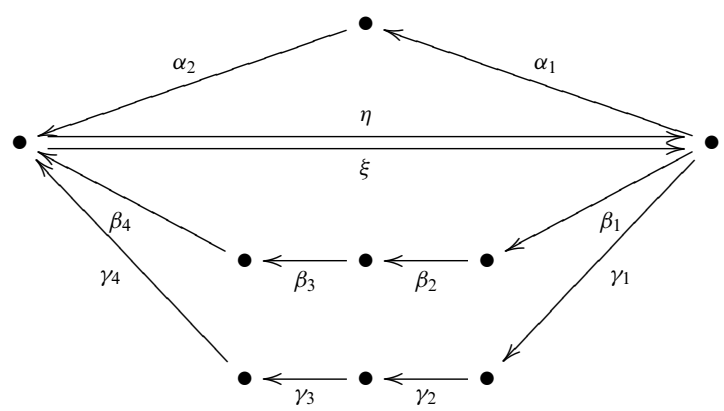

$\alpha_{1} \alpha_{2}+\beta_{1} \beta_{2} \beta_{3} \beta_{4}+\gamma_{1} \gamma_{2} \gamma_{3} \gamma_{4}=0, \eta \alpha_{1}=0, \alpha_{2} \eta=0, \xi \beta_{1}=0, \beta_{4} \xi=0, \eta \gamma_{1}=\xi \gamma_{1}$, $\gamma_{4} \eta=\gamma_{4} \xi, \beta_{2} \beta_{3} \beta_{4} \eta \beta_{1} \beta_{2}=0, \beta_{3} \beta_{4} \eta \beta_{1} \beta_{2} \beta_{3}=0, \gamma_{2} \gamma_{3} \gamma_{4} \eta \gamma_{1} \gamma_{2}=0, \gamma_{3} \gamma_{4} \eta \gamma_{1} \gamma_{2} \gamma_{3}=0$.

$\Lambda(2,3,6)$ :

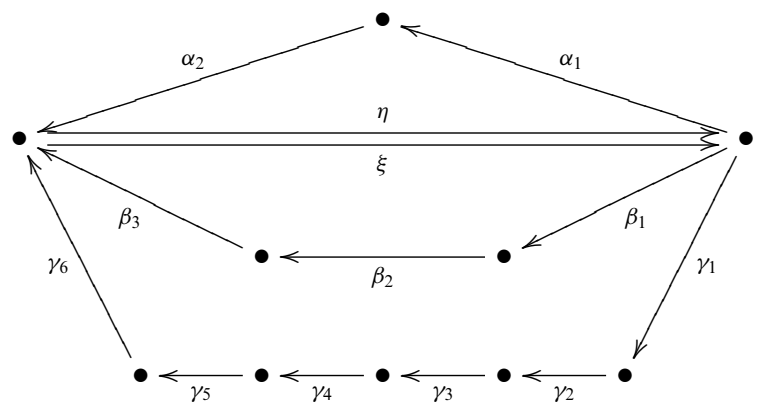

$\alpha_{1} \alpha_{2}+\beta_{1} \beta_{2} \beta_{3}+\gamma_{1} \gamma_{2} \gamma_{3} \gamma_{4} \gamma_{5} \gamma_{6}=0, \quad \eta \alpha_{1}=0, \quad \alpha_{2} \eta=0, \quad \xi \beta_{1}=0, \quad \beta_{3} \xi=0$, $\eta \gamma_{1}=\xi \gamma_{1}, \quad \gamma_{6} \eta=\gamma_{6} \xi, \quad \beta_{2} \beta_{3} \eta \beta_{1} \beta_{2}=0, \quad \gamma_{2} \gamma_{3} \gamma_{4} \gamma_{5} \gamma_{6} \eta \gamma_{1} \gamma_{2}=0, \quad \gamma_{3} \gamma_{4} \gamma_{5} \gamma_{6} \eta \gamma_{1} \gamma_{2} \gamma_{3}=0$, $\gamma_{4} \gamma_{5} \gamma_{6} \eta \gamma_{1} \gamma_{2} \gamma_{3} \gamma_{4}=0, \gamma_{5} \gamma_{6} \eta \gamma_{1} \gamma_{2} \gamma_{3} \gamma_{4} \gamma_{5}=0$.

A direct calculation shows that $\Lambda(2,2,2,2, \lambda) \cong \mathrm{T}(C(2,2,2,2, \lambda))$, $\Lambda(3,3,3) \cong \mathrm{T}(C(3,3,3)), \Lambda(2,4,4) \cong \mathrm{T}(C(2,4,4))$ and $\Lambda(2,3,6) \cong \mathrm{T}(C(2,3,6))$, 
where $C(2,2,2,2, \lambda), C(3,3,3), C(2,4,4)$ and $C(2,3,6)$ are the canonical algebras of tubular types $(2,2,2,2),(3,3,3),(2,4,4)$ and $(2,3,6)$, respectively.

The following derived equivalence classification of the standard nondomestic symmetric algebras of polynomial growth follows from [6, Theorem] (see also [19] for the derived equivalence of trivial extensions of tubular algebras) and the facts described above.

THEOREM 4.1. Let $A$ be a connected standard nondomestic symmetric algebra of polynomial growth. Then $A$ is derived equivalent to one of the following algebras:

- two simple modules: $\Lambda_{2}^{\prime}$ and $\Lambda_{3}^{\prime}(\lambda), \lambda \in K \backslash\{0,1\}$;

- three simple modules: $\Lambda_{5}^{\prime}$ and $A_{1}(\lambda), \lambda \in K \backslash\{0,1\}$;

- four simple modules: $\Lambda_{9}^{\prime}(\operatorname{char} K=2)$ and $A_{4}$;

- six simple modules: $\Lambda(2,2,2,2, \lambda), \lambda \in K \backslash\{0,1\}$;

- eight simple modules: $\Lambda(3,3,3)$;

- nine simple modules: $\Lambda(2,4,4)$,

- ten simple modules: $\Lambda(2,3,6)$.

Note that in the cases of four, eight, nine and ten simple modules, the above classification does not depend on a scalar. Moreover, for any $\lambda \in K \backslash\{0,1\}$, the centres of the algebras $\Lambda_{2}^{\prime}$ and $\Lambda_{3}^{\prime}(\lambda)$ are not isomorphic ([6, Lemma 2.2]), and hence $\Lambda_{2}^{\prime}$ and $\Lambda_{3}^{\prime}(\lambda)$ are not derived equivalent, by Corollary 2.6. Similarly, the centres of the algebras $\Lambda_{9}^{\prime}$ and $A_{4}$ are not isomorphic ([6, Lemma 4.6]), and hence $\Lambda_{9}^{\prime}$ and $A_{4}$ are not derived equivalent. Moreover, for any $\lambda \in K \backslash\{0,1\}$, the algebras $\Lambda_{5}^{\prime}$ and $A_{1}(\lambda)$ have nonisomorphic stable Auslander-Reiten quivers (see the proof of the Main Theorem), and hence are not derived equivalent, by Corollary 2.4. Therefore, the classification is complete up to the scalars $\lambda \in K \backslash\{0,1\}$ occurring in the algebras $\Lambda_{3}^{\prime}(\lambda)$ and $A_{1}(\lambda)$. We do not know how to decide for which scalars $\lambda, \mu \in K \backslash\{0,1\}$ the algebras $\Lambda_{3}^{\prime}(\lambda)$ and $\Lambda_{3}^{\prime}(\mu)$ (respectively, $A_{1}(\lambda)$ and $A_{1}(\mu)$ ) are derived equivalent.

It has been proved in [9, Theorem 1.1] that the nonstandard nondomestic symmetric algebras of polynomial growth occur only in characteristics 2 and 3 . Furthermore, the following theorem proved in [7] gives the derived equivalence classification of these algebras (when comparing with the results of [7] please note that the algebra $\Lambda_{10}$ occurring there is not symmetric and therefore does not have to be considered here).

THEOREM 4.2. Let A be a connected nonstandard nondomestic symmetric algebra of polynomial growth. Then $A$ is derived equivalent to one of the following algebras:

- two simple modules: $\Lambda_{2}(\operatorname{char} K=3)$ and $\Lambda_{3}(\lambda), \lambda \in K \backslash\{0,1\}(\operatorname{char} K=2)$;

- three simple modules: $\Lambda_{5}(\operatorname{char} K=2)$;

- four simple modules: $\Lambda_{9}(\operatorname{char} K=2)$.

The above classification is complete up to the scalars $\lambda \in K \backslash\{0,1\}$ occurring in the algebras $\Lambda_{3}(\lambda)$. We do not know how to decide for which scalars $\lambda, \mu \in K \backslash\{0,1\}$ and $K$ of characteristic 2 , the algebras $\Lambda_{3}(\lambda)$ and $\Lambda_{3}(\mu)$ are derived equivalent.

5. Proof of the Main Theorem. Let $\Lambda$ be a basic, connected, nonstandard, nondomestic, symmetric algebra of polynomial growth over $K$. Then it follows from Theorem 4.2 that either char $K=3$ and $\Lambda$ is derived equivalent to the algebra $\Lambda_{2}$ or char $K=2$ and $\Lambda$ is derived equivalent to one of the algebras $\Lambda_{3}(\lambda), \lambda \in K \backslash\{0,1\}$, $\Lambda_{5}$, or $\Lambda_{9}$. 
Assume now that $A$ is a standard self-injective algebra over $K$ which is derived equivalent to $\Lambda$. Then, by Theorems $2.1,2.2$ and $2.3, A$ is a nondomestic symmetric algebra of polynomial growth. Moreover, by Corollary 2.6, the centres $Z(A)$ and $Z(\Lambda)$ are isomorphic, and hence $A$ is connected, because $\Lambda$ is connected (by assumption on $\Lambda$ ). Since $A$ is by assumption a standard algebra, we conclude that $A$ is derived equivalent to one of the algebras listed in Theorem 4.1. Furthermore, it follows from Theorem 2.7 that the Grothendieck groups $K_{0}(\Lambda)$ and $K_{0}(A)$ are isomorphic, and hence the quivers $Q_{\Lambda}$ of $\Lambda$ and $Q_{A}$ of $A$ have the same number of vertices (see [4, II.3 and III.3]). Finally, by the general theory of self-injective algebras of tubular type (see [40, Section 5], or [39, Section 3]), we know that the stable Auslander-Reiten quivers $\Gamma_{\Lambda_{5}}^{s}$ and $\Gamma_{\Lambda_{5}^{\prime}}^{s}$ consist of $\mathbb{P}_{1}(K)$-families of stable tubes of tubular type $(2,4,4)$, while the stable Auslander-Reiten quivers $\Gamma_{A_{1}(\lambda)}^{s}, \lambda \in K \backslash\{0,1\}$, consist of $\mathbb{P}_{1}(K)$-families of stable tubes of tubular type $(2,2,2,2)$. Hence, by Corollary $2.4, \Lambda_{5}$ (respectively, $\Lambda_{5}^{\prime}$ ) is not derived equivalent to an algebra $A_{1}(\lambda), \lambda \in K \backslash\{0,1\}$.

Moreover, $\Lambda_{2}$ is not derived equivalent to $\Lambda_{3}^{\prime}(\lambda)$, since the former is of tubular type $(3,3,3)$ and the latter is of tubular type $(2,2,2,2)$. Similarly, $\Lambda_{3}(\lambda)$ is not derived equivalent to $\Lambda_{2}^{\prime}$.

Finally, $\Lambda_{9}$ is not derived equivalent to $A_{4}$ since their centres have different dimensions. In fact, the centre of $\Lambda_{9}$ is of dimension 5 , generated by the unit element 1 and the (one-dimensional) socles of the four projective indecomposable modules; on the other hand, the centre of $A_{4}$ has an additional basis element $\beta \alpha-\xi \epsilon-\gamma \delta$ and hence has dimension 6 (cf. also [6, Lemma 4.6]).

Therefore, in order to prove the Main Theorem, it remains to show that

(1) for char $K=3$, the algebras $\Lambda_{2}$ and $\Lambda_{2}^{\prime}$ are not derived equivalent;

(2) for char $K=2$ and $\lambda, \mu \in K \backslash\{0,1\}$, the algebras $\Lambda_{3}(\lambda)$ and $\Lambda_{3}(\mu)^{\prime}$ are not derived equivalent;

(3) for char $K=2$, the algebras $\Lambda_{5}$ and $\Lambda_{5}^{\prime}$ are not derived equivalent;

(4) for char $K=2$, the algebras $\Lambda_{9}$ and $\Lambda_{9}^{\prime}$ are not derived equivalent.

In order to prove (1)-(3), we will use the sequences of Külshammer ideals, as described in Section 3. To this end we have to provide below explicit associative, nondegenerate, symmetric bilinear forms on the algebras occurring in (1)-(3). It is well known that such a form exists (i.e. that an algebra $A$ is symmetric) if and only if there is a $K$-linear form $\Psi: A \rightarrow K$, called a symmetrizing form, such that $\Psi(a b)=\Psi(b a)$ for all $a, b \in A$ and the kernel of $\Psi$ does not contain any nonzero left or right ideal of $A$.

The algebras in (4) cannot be distinguished by the Külshammer ideals; for proving (4), we shall use a recent result of Al-Kadi [1] on the second Hochschild cohomology of the two algebras involved.

(1) Let $\operatorname{char} K=3$. For both of the algebras $\Lambda_{2}$ and $\Lambda_{2}^{\prime}$, a $K$-linear basis consisting of the different nonzero paths is given by

$$
\mathcal{B}=\left\{e_{1}, \alpha, \alpha^{2}, \alpha^{3}=\gamma \beta, \alpha^{4}=\alpha \gamma \beta=\gamma \beta \alpha, \gamma, \alpha \gamma, e_{2}, \beta, \beta \alpha, \beta \alpha \gamma\right\} .
$$

Also for both algebras $\Lambda=\Lambda_{2}$ and $\Lambda=\Lambda_{2}^{\prime}$, the centre is as $K$-vector space generated as follows:

$$
Z(\Lambda)=\left\langle 1, \alpha^{2}, \alpha^{3}, \alpha^{4}, \beta \alpha \gamma\right\rangle_{K}
$$


It follows from [8, Section 4] (or can be checked directly) that a symmetrizing form $\Psi^{\prime}$ on the standard algebra $\Lambda_{2}^{\prime}$ is given by assigning 1 to $\alpha^{4}$ and $\beta \alpha \gamma$, and 0 to all other elements of the basis $\mathcal{B}$.

Note that we cannot use the same form for the nonstandard algebra $\Lambda_{2}$, since $\Psi^{\prime}(\gamma \beta)=0$ whereas $\Psi^{\prime}(\beta \gamma)=\Psi^{\prime}(\beta \alpha \gamma)=1$, i.e. the form is not symmetric. Instead, a symmetrizing form $\Psi$ on $\Lambda_{2}$ is given by assigning 1 to $\alpha^{4}$, to $\beta \alpha \gamma$ and to $\alpha^{3}=\gamma \beta$, and assigning 0 to all other elements of the basis $\mathcal{B}$.

Now we have to examine the commutator subspaces.

First, we consider the standard algebra $\Lambda_{2}^{\prime}$. Since $\beta \gamma=0$, we obtain

$$
K\left(\Lambda_{2}^{\prime}\right)=\langle\gamma, \beta, \alpha \gamma, \beta \alpha, \gamma \beta, \beta \alpha \gamma-\alpha \gamma \beta\rangle_{K}
$$

In particular, $\alpha^{3}=\gamma \beta \in K\left(\Lambda_{2}^{\prime}\right)$ from which it follows that

$$
T_{1}\left(\Lambda_{2}^{\prime}\right):=\left\{x \in \Lambda_{2}^{\prime} \mid x^{3} \in K\left(\Lambda_{2}^{\prime}\right)\right\}=J\left(\Lambda_{2}^{\prime}\right),
$$

where $J\left(\Lambda_{2}^{\prime}\right)$ denotes the ideal generated by the arrows of the quiver. (In fact, the basis elements other than $\alpha$ in $J\left(\Lambda_{2}^{\prime}\right)$ clearly belong to $T_{1}\left(\Lambda_{2}^{\prime}\right)$ since their third powers vanish.)

For the first Külshammer ideal, we therefore get

$$
T_{1}\left(\Lambda_{2}^{\prime}\right)^{\perp}=\left(J\left(\Lambda_{2}^{\prime}\right)\right)^{\perp}=\operatorname{soc}\left(\Lambda_{2}^{\prime}\right),
$$

that is, the sequence of Külshammer ideals for $\Lambda_{2}^{\prime}$ has length 2 .

Secondly, we consider the nonstandard algebra $\Lambda_{2}$. Now, $\beta \gamma=\beta \alpha \gamma$ is nonzero, and the commutator subspace is

$$
K\left(\Lambda_{2}\right)=\langle\gamma, \beta, \alpha \gamma, \beta \alpha, \gamma \beta-\beta \alpha \gamma, \beta \alpha \gamma-\alpha \gamma \beta\rangle_{K}
$$

In particular, $\alpha \notin T_{1}\left(\Lambda_{2}\right):=\left\{x \in \Lambda_{2} \mid x^{3} \in K\left(\Lambda_{2}\right)\right\}$. But then $\alpha^{3} \in Z\left(\Lambda_{2}\right)$ is orthogonal (with respect to the form $\Psi$ above) to all elements in $T_{1}\left(\Lambda_{2}\right)$. Thus, the sequence of Külshammer ideals takes the form

$$
\operatorname{soc}\left(\Lambda_{2}\right) \underbrace{\subset}_{1} T_{1}\left(\Lambda_{2}\right)^{\perp}=\left\langle\alpha^{3}, \alpha^{4}, \beta \alpha \gamma\right\rangle_{K} \underbrace{\subset}_{2} Z\left(\Lambda_{2}\right),
$$

where the numbers under the inclusion signs denote codimensions. Since the sequences of Külshammer ideals for the algebras $\Lambda_{2}^{\prime}$ and $\Lambda_{2}$ have different lengths (and different codimensions), we can conclude from Theorem 3.1 that $\Lambda_{2}^{\prime}$ and $\Lambda_{2}$ are not derived equivalent.

(2) Let char $K=2$ and $\lambda, \mu \in K \backslash\{0,1\}$. For both types of algebras $\Lambda_{3}(\lambda)$ and $\Lambda_{3}(\mu)^{\prime}$, a $K$-linear basis consisting of the different nonzero paths is given by

$$
\mathcal{B}=\left\{e_{1}, e_{2}, \alpha, \beta, \sigma, \gamma, \alpha^{2}, \alpha \sigma, \beta^{2}, \gamma \alpha, \alpha^{3}, \beta^{3}\right\}
$$

In fact, note that for $\Lambda_{3}(\mu)^{\prime}$ we have that $\alpha^{4}=(\sigma \gamma)^{2}=\mu \sigma \beta^{2} \gamma=\mu \alpha \sigma \gamma \alpha=$ $\mu \alpha^{4}$, from which $\alpha^{4}=0$ follows since $\mu \neq 1$. Similarly, one checks that $\gamma \alpha^{2}=$ $\beta \gamma \alpha=\gamma \sigma \gamma=0$ and $\alpha^{2} \sigma=\alpha \sigma \beta=\sigma \gamma \sigma=0$ in $\Lambda_{3}(\mu)^{\prime}$, and that $\beta^{4}=0$ in $\Lambda_{3}(\lambda)$ and $\Lambda_{3}(\mu)^{\prime}$. 
For both types of algebras $\Lambda=\Lambda_{3}(\lambda)$ and $\Lambda=\Lambda_{3}^{\prime}(\mu)$, the centre has a basis of the form

$$
Z(\Lambda)=\left\langle 1, \alpha+\beta, \alpha^{2}, \beta^{2}, \alpha^{3}, \beta^{3}\right\rangle_{K}
$$

It follows from [8, Section 4] (or can be checked directly) that a symmetrizing form $\Psi^{\prime}$ on the standard algebra $\Lambda_{3}(\mu)^{\prime}$ is given by assigning 1 to $\alpha^{3}, \mu^{-1}$ to $\beta^{3}$, and 0 to all remaining elements of the basis $\mathcal{B}$.

Note that we cannot use the same form for the nonstandard algebra $\Lambda_{3}(\lambda)$, because $0=\Psi^{\prime}\left(\alpha^{2}\right)=\Psi^{\prime}\left(\sigma \gamma+\alpha^{3}\right)=0+1=1$. Instead, a symmetrizing form $\Psi$ for $\Lambda_{3}(\lambda)$ is given by assigning 1 to $\alpha^{3}$ and to $\alpha^{2}, \lambda^{-1}$ to $\beta^{3}$, and 0 to all remaining elements of the basis $\mathcal{B}$.

The commutator subspaces for both $\Lambda=\Lambda_{3}(\lambda)$ and $\Lambda=\Lambda_{3}(\mu)^{\prime}$ are generated as vector space as follows:

$$
K(\Lambda)=\langle\sigma, \gamma, \alpha \sigma, \gamma \alpha, \gamma \sigma-\sigma \gamma, \gamma \sigma \beta-\sigma \beta \gamma\rangle_{K}
$$

Note that the only crucial difference in the relations is that $\alpha^{2}=\sigma \gamma$ for the standard algebras $\Lambda_{3}^{\prime}(\mu)$, whereas $\alpha^{2}=\sigma \gamma+\alpha^{3}$ for the nonstandard algebras $\Lambda_{3}(\lambda)$. This means that the element $\gamma \sigma-\sigma \gamma$ in the commutator space equals $\mu \beta^{2}-\alpha^{2}$ for $\Lambda_{3}^{\prime}(\mu)$, and $\lambda \beta^{2}-\alpha^{2}+\alpha^{3}$ for $\Lambda_{3}(\lambda)$, respectively. This has consequences for the spaces $T_{1}(\Lambda)=\left\{x \in \Lambda \mid x^{2} \in K(\Lambda)\right\}$. Namely, let $\sqrt{\mu}$ be the unique square root of $\mu$ in $K$ (recall that $K$ is algebraically closed, and of characteristic 2). Then we get for the standard algebras that $\alpha+\sqrt{\mu} \beta \in T_{1}\left(\Lambda_{3}^{\prime}(\mu)\right)$, whereas there is no analogous element in $T_{1}\left(\Lambda_{3}(\lambda)\right)$. More precisely, we have

$$
T_{1}\left(\Lambda_{3}^{\prime}(\mu)\right)=\left\langle\alpha+\sqrt{\mu} \beta, \sigma, \gamma, \alpha^{2}, \alpha \sigma, \beta^{2}, \gamma \alpha, \alpha^{3}, \beta^{3}\right\rangle_{K}
$$

and

$$
T_{1}\left(\Lambda_{3}(\lambda)\right)=\left\langle\sigma, \gamma, \alpha^{2}, \alpha \sigma, \beta^{2}, \gamma \alpha, \alpha^{3}, \beta^{3}\right\rangle_{K}
$$

From this we can determine the first Külshammer ideals and their codimensions as follows. For the standard algebras, we obtain (using the symmetrizing form $\Psi^{\prime}$ above)

$$
\operatorname{soc}\left(\Lambda_{3}^{\prime}(\mu)\right) \underbrace{\subset}_{1} T_{1}\left(\Lambda_{3}^{\prime}(\mu)\right)^{\perp}=\left\langle\alpha^{2}+\sqrt{\mu} \beta^{2}, \alpha^{3}, \beta^{3}\right\rangle_{K} \underbrace{\subset}_{3} Z\left(\left(\Lambda_{3}^{\prime}(\mu)\right) .\right.
$$

In fact, $\Psi^{\prime}\left(\left(\alpha^{2}+\sqrt{\mu} \beta^{2}\right)(\alpha+\sqrt{\mu} \beta)\right)=\Psi^{\prime}\left(\alpha^{3}+\mu \beta^{3}\right)=1+\mu \mu^{-1}=0$ since char $K=2$; on the other hand, $\alpha^{2} \notin T_{1}\left(\Lambda_{3}^{\prime}(\mu)\right)^{\perp}$ because $\alpha+\sqrt{\mu} \beta \in$ $T_{1}\left(\Lambda_{3}^{\prime}(\mu)\right)$, similarly for $\beta^{2}$.

For the nonstandard algebras, we get (using the symmetrizing form $\Psi$ above)

$$
\operatorname{soc}\left(\Lambda_{3}(\lambda)\right) \underbrace{\subset}_{2} T_{1}\left(\Lambda_{3}^{\prime}(\lambda)\right)^{\perp}=\left\langle\alpha^{2}, \beta^{2}, \alpha^{3}, \beta^{3}\right\rangle_{K} \underbrace{\subset}_{2} Z\left(\left(\Lambda_{3}(\lambda)\right) .\right.
$$

Because of the different codimensions we can again conclude by Theorem 3.1 that $\Lambda_{3}(\lambda)$ and $\Lambda_{3}^{\prime}(\mu)$ are not derived equivalent, as claimed. 
(3) Let char $K=2$. For both of the algebras $\Lambda_{5}$ and $\Lambda_{5}^{\prime}$, a $K$-linear basis consisting of the different nonzero paths is given by

$$
\mathcal{B}=\left\{e_{1}, e_{2}, e_{3}, \alpha, \beta, \gamma, \delta, \sigma, \alpha \gamma, \alpha^{2}=\gamma \beta, \beta \alpha, \alpha^{3}=\delta \sigma, \sigma \delta, \beta \alpha \gamma\right\} .
$$

Also for both of the algebras $\Lambda=\Lambda_{5}$ and $\Lambda=\Lambda_{5}^{\prime}$, the centre is as vector space generated as follows:

$$
Z(\Lambda)=\left\langle 1, \alpha^{2}, \alpha^{3}, \sigma \delta, \beta \alpha \gamma\right\rangle_{K}
$$

It follows from [8, Section 4] (or can be checked directly) that a symmetrizing form $\Psi^{\prime}$ on the standard algebra $\Lambda_{5}^{\prime}$ is given by assigning 1 to the socle elements $\alpha^{3}, \beta \alpha \gamma$ and $\sigma \delta$, and assigning 0 to all other elements of the basis $\mathcal{B}$.

Note that one cannot use the same symmetrizing form for the nonstandard algebra $\Lambda_{5}$ since $\Psi^{\prime}(\gamma \beta)=0 \neq 1=\Psi^{\prime}(\beta \alpha \gamma)=\Psi^{\prime}(\beta \gamma)$, i.e. the form is not symmetric on $\Lambda_{5}$.

In fact, a symmetrizing form $\Psi$ for $\Lambda_{5}$ is given by assigning 1 to $\alpha^{3}, \beta \alpha \gamma, \sigma \delta$ and also to $\alpha^{2}$, and assigning 0 to all other elements of the basis $\mathcal{B}$.

We shall need the commutator subspaces. It turns out that the only crucial difference comes from the fact that $\beta \gamma=0$ in $\Lambda_{5}^{\prime}$, whereas $\beta \gamma=\beta \alpha \gamma$ is nonzero in $\Lambda_{5}$. Hence, we get

$$
K(\Lambda)=\langle\beta, \gamma, \delta, \sigma, \beta \alpha, \alpha \gamma, \delta \sigma-\sigma \delta, \beta \alpha \gamma-\alpha \gamma \beta, X\rangle_{K},
$$

where $X=\gamma \beta$ for $\Lambda_{5}^{\prime}$, and $X=\gamma \beta-\beta \gamma$ for $\Lambda_{5}$, respectively. This has the following implication for $T_{1}(\Lambda):=\left\{x \in \Lambda \mid x^{2} \in K(\Lambda)\right\}$. Namely, since $\alpha^{2}=$ $\gamma \beta$, we have $\alpha \in T_{1}\left(\Lambda_{5}^{\prime}\right)$, but $\alpha \notin T_{1}\left(\Lambda_{5}\right)$. More precisely, denoting by $J(\Lambda)$ the ideal generated by the arrows of the quiver, we obtain

$$
T_{1}\left(\Lambda_{5}^{\prime}\right)=J\left(\Lambda_{5}^{\prime}\right)
$$

whereas

$$
T_{1}\left(\Lambda_{5}\right) \subset J\left(\Lambda_{5}\right)
$$

has codimension 1. (In fact, it is easy to see that all other basis elements of the ideal $J(\Lambda)$ are contained in $T_{1}(\Lambda)$.)

Now we examine the first Külshammer ideals $T_{1}(\Lambda)^{\perp}$. First we consider the standard algebra $\Lambda_{5}^{\prime}$. Here we get

$$
T_{1}\left(\Lambda_{5}^{\prime}\right)^{\perp}=\left(J\left(\Lambda_{5}^{\prime}\right)\right)^{\perp}=\operatorname{soc}\left(\Lambda_{5}^{\prime}\right)=\left\langle\alpha^{3}, \sigma \delta, \beta \alpha \gamma\right\rangle_{K} .
$$

In fact, $\alpha^{2} \notin T_{1}\left(\Lambda_{5}^{\prime}\right)^{\perp}$ since $\alpha \in T_{1}\left(\Lambda_{5}^{\prime}\right)$ and $\Psi^{\prime}\left(\alpha^{2} \cdot \alpha\right)=\Psi^{\prime}\left(\alpha^{3}\right)=1$.

On the other hand, for the nonstandard algebra $\Lambda_{5}$, we have that $\alpha^{2}$ is orthogonal to all elements in $T_{1}\left(\Lambda_{5}\right)$ since $\alpha \notin T_{1}\left(\Lambda_{5}\right)$ (and $\alpha^{2} \cdot w=0$ for every arrow $w \neq \alpha$ in the quiver), that is,

$$
T_{1}\left(\Lambda_{5}\right)^{\perp}=\left\langle\alpha^{2}, \alpha^{3}, \sigma \delta, \beta \alpha \gamma\right\rangle_{K} .
$$

Since the first Külshammer ideals have different codimensions inside the centre, we can conclude by Theorem 3.1 that $\Lambda_{5}$ and $\Lambda_{5}^{\prime}$ are not derived equivalent, as claimed. 
(4) Let char $K=2$. The algebras $\Lambda_{9}$ and $\Lambda_{9}^{\prime}$ cannot be distinguished by their Külshammer ideals. Instead we shall use Hochschild cohomology. It has recently been shown by Al-Kadi ([1], theorems 3.1 and 4.1) that

$$
\operatorname{dim}_{K} H H^{2}\left(\Lambda_{9}\right)=4 \text { and } \operatorname{dim}_{K} H H^{2}\left(\Lambda_{9}^{\prime}\right)=3 .
$$

Therefore, applying Theorem 2.5, we conclude ([1], corollary 4.2) that $\Lambda_{9}$ and $\Lambda_{9}^{\prime}$ are not derived equivalent.

This completes the proof of the Main Theorem.

ACKNOWLedgement. T. H. is supported by the research grant HO 1880/4-1 of the Deutsche Forschungsgemeinschaft (DFG), in the framework of the Research Priority Program SPP 1388 Representation Theory. A. S. is supported by the research grant No. N N 201269135 of the Polish Ministry of Science and Higher Education.

\section{REFERENCES}

1. D. Al-Kadi, Distinguishing derived equivalence classes using the second Hochschild cohomology group, Colloq. Math. (to appear).

2. S. Al-Nofayee, Derived equivalence for selfinjective algebras and $t$-structures, $\mathrm{PhD}$ Thesis (Bristol University, 2004).

3. H. Asashiba, The derived equivalence classification of representation-finite selfinjective algebras, J. Algebra 214 (1999), 182-221.

4. I. Assem, D. Simson and A. Skowroński, Elements of the Representation Theory of Associative Algebras 1: Techniques of Representation Theory (London Mathematical Society Student Texts vol. 65) (Cambridge University Press, Cambridge, 2006).

5. J. Białkowski, K. Erdmann and A. Skowroński, Deformed preprojective algebras of generalized Dynkin type, Trans. Amer. Math. Soc. 359 (2007), 2625-2650.

6. J. Białkowski, T. Holm and A. Skowroński, Derived equivalences for tame weakly symmetric algebras having only periodic modules, J. Algebra 269 (2003), 652-668.

7. J. Białkowski, T. Holm and A. Skowroński, On nonstandard tame selfinjective algebras having only periodic modules, Colloq. Math. 97 (2003), 33-47.

8. J. Białkowski and A. Skowroński, On tame weakly symmetric algebras algebras having only periodic modules, Arch. Math. 81 (2003), 142-154.

9. J. Białkowski and A. Skowroński, Socle deformations of selfinjective algebras of tubular type, J. Math. Soc. Japan 56 (2004), 687-716.

10. R. Bocian, T. Holm and A. Skowroński, Derived equivalence classification of weakly symmetric algebras of Euclidean type, J. Pure Appl. Algebra 191 (2004), 43-74.

11. R. Bocian, T. Holm and A. Skowronski, Derived equivalence classification of nonstandard algebras of domestic type, Comm. Alg. 35 (2007), 515-526.

12. Y. A. Drozd, Tame and wild matrix problems, in Representation Theory II, (Lecture Notes in Mathematics vol. 832) (Springer, Berlin, 1980), 242-258.

13. K. Erdmann, Blocks of Tame Representation Type and Related Algebras, (Lecture Notes in Mathematics vol. 1428) (Springer: Berlin, 1990).

14. K. Erdmann and A. Skowroński, Classification of tame symmetric algebras with periodic modules (in preparation).

15. K. Erdmann and A. Skowroński, The stable Calabi-Yau dimension of tame symmetric algebras, J. Math. Soc. Japan 58 (2006), 97-128.

16. M. Gerstenhaber, The cohomology structure of an associative ring, Ann. Math. 78(2), (1963), 267-288.

17. D. Happel, On the derived category of a finite-dimensional algebra, Comment. Math. Helv. 62 (1987), 339-389.

18. D. Happel, Triangulated Categories in the Representation Theory of Finite Dimensional Algebras, London Math. Soc. (Lecture Note Series vol. 119) (Cambridge University Press, Cambridge, 1988). 
19. D. Happel and C. M. Ringel, The derived category of a tubular algebra, in Representation Theory I. Finite Dimensional Algebras, (Lecture Notes in Mathematics vol. 1177) (Springer: Berlin, 1986), 156-180.

20. L. Héthelyi, E. Horváth, B. Külshammer and J. Murray, Central ideals and Cartan invariants of symmetric algebras, J. Algebra 293 (2005), 243-260.

21. T. Holm, Derived equivalence classification of algebras of dihedral, semidihedral, and quaternion type, J. Algebra 211 (1999), 159-205.

22. T. Holm and A. Skowroński, Derived equivalence classification of symmetric algebras of domestic type, J. Math. Soc. Japan 58 (2006), 1133-1149.

23. T. Holm and A. Zimmermann, Generalized Reynolds ideals and derived equivalences for algebras of dihedral and semidihedral type, J. Algebra 320 (2008), 3425-3437.

24. H. Krause and G. Zwara, Stable equivalence and generic modules, Bull. London Math. Soc. 32 (2000), 615-618.

25. B. Külshammer, Bemerkungen über die Gruppenalgebra als symmetrische Algebra I, J. Algebra 72 (1981), 1-7.

26. B. Külshammer, Bemerkungen über die Gruppenalgebra als symmetrische Algebra II, J. Algebra 75 (1982), 59-69.

27. B. Külshammer, Bemerkungen über die Gruppenalgebra als symmetrische Algebra III, J. Algebra 88 (1984), 279-291.

28. B. Külshammer, Bemerkungen über die Gruppenalgebra als symmetrische Algebra IV, J. Algebra 93 (1985), 310-323.

29. B. Külshammer, Group-theoretical descriptions of ring theoretical invariants of group algebras, Prog. Math. 95 (1991), 425-441.

30. T. Nakayama, On Frobeniusean algebras I, Ann. Math. 40 (1939), 611-633.

31. T. Nakayama, On Frobeniusean algebras II, Ann. of Math. 42 (1941), 1-21.

32. J. Nehring and A. Skowroński, Polynomial growth trivial extensions of simply connected algebras, Fund. Math. 132 (1989), 117-134.

33. J. Rickard, Morita theory for derived categories, J. London Math. Soc. 39 (1989), 436456.

34. J. Rickard, Derived categories and stable equivalence, J. Pure Appl. Algebra 61 (1989), 303-317. $37-48$

35. J. Rickard, Derived equivalences as derived functors, J. London Math. Soc. 43 (1991),

36. D. Simson and A. Skowroński, Elements of the Representation Theory of Associative Algebras 2: Tubes and Concealed Algebras of Euclidean Type, (London Mathematical Society Student Texts vol. 71) (Cambridge University Press, Cambridge, 2007).

37. D. Simson and A. Skowroński, Elements of the Representation Theory of Associative Algebras 3: Representation-Infinite Tilted Algebras, (London Mathematical Society Student Texts vol. 72) (Cambridge University Press, Cambridge, 2007).

38. A. Skowroński, Classification of self-injective algebras of polynomial growth (in preparation).

39. A. Skowroński, Selfinjective algebras of polynomial growth, Math. Ann. 285 (1989), 177-199.

40. A. Skowroński, Selfinjective algebras: finite and tame type, in Trends in Representation Theory of Algebras and Related Topics, Contemporary Mathematics, vol. 406, (American Mathematical Society, Providence, RI, 2006), 169-238.

41. K. Yamagata, Frobenius algebras, in Handbook of Algebra, vol. 1 (Elsevier Science B.V., Amsterdam, 1996), 841-887.

42. A. Zimmermann, Invariance of generalized Reynolds ideals under derived equivalences, Math. Proc. R. Ir. Acad. 107 (2007), 1-9. 